\title{
Optimality and duality results for a nondifferentiable multiobjective fractional programming problem
}

\author{
Ramu Dubey', Shiv K Gupta ${ }^{1 *}$ and Meraj Ali Khan²
}

\section{"Correspondence:} skgiitr@gmail.com

'Department of Mathematics, Indian Institute of Technology, Roorkee, 247 667, India Full list of author information is available at the end of the article

MSC: 90C26; 90C30; 90C32; 90C46

Keywords: duality results; multiobjective programming problem; support function; KKT conditions; efficient solution

\section{Introduction}

Higher-order duality is significant due to its computational importance as it provides higher bounds whenever an approximation is used. By introducing two different functions, $h: R^{n} \times R^{n} \rightarrow R$ and $k: R^{n} \times R^{n} \rightarrow R^{m}$, Mangasarian [1] formulated a higher-order dual for a nonlinear optimization problem, $\{\min f(x)$, subject to $g(x) \leq 0\}$. Inspired by this concept, many researchers have worked in this direction. Chen [2] has formulated higherorder multiobjective symmetric dual programs and established duality relations under higher-order $F$-convexity assumptions. A higher-order vector optimization problem and its dual have been studied by Kassem [3].

In the last several years, various optimality and duality results have been obtained for multiobjective fractional programming problems. In Chen [2], multiobjective fractional problem and its duality theorems have been considered under higher-order $(F, \alpha, \rho, d)$ convexity. Later on, Suneja et al. [4] discussed higher-order Mond-Weir and Schaible type nondifferentiable dual programs and their duality theorems under higher-order $(F, \rho, \sigma)$ type $I$-assumptions. Recently, Ying [5] has studied higher-order multiobjective symmetric fractional problem and formulated its Mond-Weir type dual. Further, duality results are obtained under higher-order $(F, \alpha, \rho, d)$-convexity.

\begin{abstract}
The purpose of this paper is to study a class of nondifferentiable multiobjective fractional programming problems in which every component of objective functions contains a term involving the support function of a compact convex set. For a $(C, \alpha, \gamma, \rho, d)$-convex function. A nontrivial example is also constructed which is in this optimality conditions for an efficient solution of the nondifferentiable multiobjective fractional programming problem are established. Further, a higher-order Mond-Weir type dual is formulated for this problem and appropriate duality results are proved der higher-order $(C, \alpha, \gamma, \rho, d)$-assumptions
\end{abstract}


Yuan et al. [6] introduced a class of functions called $(C, \alpha, \rho, d)$-convex functions and derived duality theorems for a nondifferentiable minimax fractional programming problem under $(C, \alpha, \rho, d)$-convexity. Chinchuluun et al. [7] later studied nonsmooth multiobjective fractional programming problems in the framework of $(C, \alpha, \rho, d)$-convexity.

In this paper, we first introduce the definition of higher-order $(C, \alpha, \gamma, \rho, d)$-convex with respect to a differentiable function $H: X \times R^{n} \longrightarrow R\left(X \subset R^{n}\right), p, s \in R^{n}$ and construct a nontrivial example which is higher-order $(C, \alpha, \gamma, \rho, d)$-convex but not a $(F, \alpha, \gamma, \rho, d)$ convex function. We prove that the ratio of higher-order $(C, \alpha, \gamma, \rho, d)$-convex functions is also higher-order $(\bar{C}, \bar{\alpha}, \bar{\gamma}, \bar{\rho}, \bar{d})$-convex. A sufficient optimality condition related to the efficient solution of a multiobjective nondifferentiable fractional problem has been established. Finally, we formulate a higher-order Mond-Weir type dual problem corresponding to the multiobjective nondifferentiable fractional programming problem and established usual duality relations under the aforesaid assumptions.

\section{Preliminaries}

Definition 2.1 A function $C: X \times X \times R^{n} \longrightarrow R\left(X \subset R^{n}\right)$ is said to be convex on $R^{n}$ iff, for any fixed $(x, u) \in X \times X$ and for any $x_{1}, x_{2} \in R^{n}$,

$$
C_{x, u}\left(\lambda x_{1}+(1-\lambda) x_{2}\right) \leq \lambda C_{x, u}\left(x_{1}\right)+(1-\lambda) C_{x, u}\left(x_{2}\right), \quad \forall \lambda \in(0,1)
$$

We now introduce the definition of higher-order $(C, \alpha, \gamma, \rho, d)$-convex function. Let $C$ be a convex function with respect to the third variable such that $C_{x, u}(0)=0, \forall(x, u) \in X \times X$. Let $H: X \times R^{n} \rightarrow R, \phi: X \rightarrow R$ be differentiable functions on $X$. Assume that $\alpha, \gamma: X \times X \rightarrow$ $R_{+} \backslash\{0\}, \rho \in R, d: X \times X \rightarrow R_{+}$satisfying $d\left(x, x_{0}\right)=0 \Leftrightarrow x=x_{0}$ and $p, s \in R^{n}$.

Definition 2.2 The function $\phi$ is said to be higher-order (strictly) $(C, \alpha, \gamma, \rho, d)$-convex at $u$ with respect to $H, p$ and $s$ if for each $x \in X$,

$$
\begin{aligned}
\frac{1}{\alpha(x, u)}[\phi(x)-\phi(u)] \geq & (>) C_{x, u}\left(\nabla \phi(u)+\nabla_{p} H(u, p)\right) \\
& +\frac{1}{\gamma(x, u)}\left[H(u, s)-s^{T} \nabla_{s} H(u, s)\right]+\frac{\rho d(x, u)}{\alpha(x, u)} .
\end{aligned}
$$

Remark 2.1 A differentiable function $f=\left(f_{1}, f_{2}, \ldots, f_{k}\right): X \rightarrow R^{k}$ is $(C, \alpha, \gamma, \rho, d)$-convex if for all $i=1,2, \ldots, k, f_{i}$ is $\left(C, \alpha_{i}, \gamma_{i}, \rho_{i}, d_{i}\right)$-convex.

\section{Remark 2.2}

(i) If $H(u, \cdot)=0$, then Definition 2.2 becomes that of a $(C, \alpha, \rho, d)$-convex function as given in [6, 8]. Further if $\alpha(x, u)=1, \rho=0$, and $C_{x, u}(a)=\eta^{T}(x, u) a$, for $\eta: X \times X \rightarrow R^{n}$, then $(C, \alpha, \gamma, \rho, d)$-convexity reduces to invexity (see Hanson [9]).

(ii) If $H(u, \cdot)=\frac{1}{2}(\cdot)^{T} \nabla^{2} f(u)(\cdot), \alpha(x, u)=\gamma(x, u)$, and $p=s$, then Definition 2.2 reduces to the first expression of a second-order $(C, \alpha, \rho, d)$-type I-convex function given in Gupta et al. [10].

(iii) If $\alpha(x, u)=\gamma(x, u)=1, \rho=0, H(u, \cdot)=\frac{1}{2}(\cdot)^{T} \nabla^{2} f(u)(\cdot), p=s$, and $C_{x, u}(a)=\eta^{T}(x, u) a$, where $\eta: X \times X \rightarrow R^{n}$, the above definition becomes that of $\eta$-bonvexity introduced by Pandey [11]. 
(iv) If $C$ is sublinear with respect to third variable and $p=s$, then the above definition reduces to higher-order $(F, \alpha, \gamma, \rho, d)$-convexity as given in Gulati and Saini [12]. Furthermore, if $\gamma(x, u)=\alpha(x, u)$, then Definition 2.2 reduces to higher-order $(F, \alpha, \rho, d)$-convexity as in Ying [5]. Moreover, if $H(u, \cdot)=0$, then Definition 2.2 becomes that of a $(F, \alpha, \rho, d)$-convex function as introduced by Liang et al. [13].

Remark 2.3 Every $(F, \alpha, \gamma, \rho, d)$-convex function is $(C, \alpha, \gamma, \rho, d)$-convex. However, the converse need not be true. This is illustrated by the following example.

Example 2.1 Let $X=\{x: x \geq 1\} \subset R, f: X \rightarrow R, C: X \times X \times R \rightarrow R, H: X \times R \rightarrow R$, and $d: X \times X \rightarrow R_{+}$be defined as

$$
f(x)=\frac{x^{2}+x+2}{x+1}, \quad C_{x, u}(a)=a^{2}(x-u)^{2}, \quad H(u, \cdot)=\frac{(\cdot)}{u+1}, \quad d(x, u)=(x-u)^{2}
$$

Clearly, the function $C$ (defined above) is convex with respect to the third variable satisfying $C_{x, u}(0)=0, \forall x, u \in X$. Also, $d(x, u)=0 \Leftrightarrow x=u$.

Let $\rho=-1$ and $\alpha=\gamma=\frac{3}{4}$. Then at $u=1$, for all $x \in X$, we have

$$
\begin{aligned}
\frac{1}{\alpha(x, u)}[f(x)-f(u)]= & \frac{4}{3}\left(\frac{x^{2}-x}{x+1}\right) \geq C_{x, u}\left(\nabla_{x} f(u)+\nabla_{p} H(u, p)\right) \\
& +\frac{1}{\gamma(x, u)}\left[H(u, s)-s^{T} \nabla_{s} H(u, s)\right]+\frac{\rho d(x, u)}{\alpha(x, u)}=-\frac{1}{3}(x-1)^{2} .
\end{aligned}
$$

Hence, $f$ is a higher-order $(C, \alpha, \gamma, \rho, d)$-convex function with respect to $H, p$, and $s$. But considering the same $C, f$ is not higher-order $(F, \alpha, \gamma, \rho, d)$-convex because $C$ is not a sublinear functional with respect to the third variable.

Definition 2.3 [14] Let $C$ be a compact convex set in $R^{n}$. The support function of $C$ is defined by

$$
S(x \mid C)=\max \left\{x^{T} y: y \in C\right\}
$$

\section{Problem formulation and optimality conditions}

Consider the following nondifferentiable multiobjective programming problem:

(MFP) Minimize $F(x)=\left\{\frac{f_{1}(x)+S\left(x \mid C_{1}\right)}{g_{1}(x)-S\left(x \mid D_{1}\right)}, \frac{f_{2}(x)+S\left(x \mid C_{2}\right)}{g_{2}(x)-S\left(x \mid D_{2}\right)}, \ldots, \frac{f_{k}(x)+S\left(x \mid C_{k}\right)}{g_{k}(x)-S\left(x \mid D_{k}\right)}\right\}$ subject to $x \in X^{0}=\left\{x \in X \subset R^{n}: h_{j}(x)+S\left(x \mid E_{j}\right) \leq 0, j=1,2, \ldots, m\right\}$,

where $f_{i}, g_{i}: X \rightarrow R(i=1,2, \ldots, k)$ and $h_{j}: X \rightarrow R(j=1,2, \ldots, m)$ are continuously differentiable functions. Assume that $f_{i}(\cdot)+S\left(\cdot \mid C_{i}\right) \geq 0$ and $g_{i}(\cdot)-S\left(\cdot \mid D_{i}\right)>0 ; C_{i}, D_{i}$, and $E_{j}$ are compact convex sets in $R^{n}$ and $S\left(x \mid C_{i}\right), S\left(x \mid D_{i}\right)$, and $S\left(x \mid E_{j}\right)$ denote the support functions of compact convex sets, $C_{i}, D_{i}$, and $E_{j}, i=1,2, \ldots, k, j=1,2, \ldots, m$, respectively.

Definition 3.1 [15] A point $x^{0} \in X^{0}$ is a weakly efficient solution of (MFP), if there exists no $x \in X^{0}$ such that for every $i=1,2, \ldots, k, \frac{f_{i}(x)+S\left(x \mid C_{i}\right)}{g_{i}(x)-S\left(x \mid D_{i}\right)}<\frac{f_{i}\left(x^{0}\right)+S\left(x^{0} \mid C_{i}\right)}{g_{i}\left(x^{0}\right)-S\left(x^{0} \mid D_{i}\right)}$. 
Definition 3.2 [16] A point $x^{0} \in X^{0}$ is an efficient solution (or a Pareto optimal solution) of (MFP), if there exists no $x \in X^{0}$ such that for every $i=1,2, \ldots, k, \frac{f_{i}(x)+S\left(x \mid C_{i}\right)}{g_{i}(x)-S\left(x \mid D_{i}\right)} \leq \frac{f_{i}\left(x^{0}\right)+S\left(x^{0} \mid C_{i}\right)}{g_{i}\left(x^{0}\right)-S\left(x^{0} \mid D_{i}\right)}$ and for some $r=1,2, \ldots, k, \frac{f_{r}(x)+S\left(x \mid C_{r}\right)}{g_{r}(x)-S\left(x \mid D_{r}\right)}<\frac{f_{r}\left(x^{0}\right)+S\left(x^{0} \mid C_{r}\right)}{g_{r}\left(x^{0}\right)-S\left(x^{0} \mid D_{r}\right)}$.

Lemma 3.1 [17] If for a given $\bar{\lambda}_{i}>0\left(\bar{\lambda}_{i} \geq 0\right.$ and $\left.\bar{\lambda} \neq 0\right), i=1,2, \ldots, k, \bar{x} \in X$ is an optimal solution for the following single-objective problem:

$$
\begin{gathered}
\left(\mathbf{F} \mathbf{P}_{\lambda}\right) \text { minimize } G(x)=\sum_{i=1}^{k} \bar{\lambda}_{i}\left(\frac{f_{i}(x)+S\left(x \mid C_{i}\right)}{g_{i}(x)-S\left(x \mid D_{i}\right)}\right) \\
\text { subject to } x \in X \subset R^{n},
\end{gathered}
$$

then $\bar{x}$ is an efficient solution (a weakly efficient solution) for (MFP).

Theorem 3.1 For some $t$, assume $f_{t}(\cdot)+(\cdot)^{T} z_{t}$ and $-\left(g_{t}(\cdot)-(\cdot)^{T} v_{t}\right)$ are $\left(C, \alpha_{t}, \gamma_{t}, \rho_{t}, d_{t}\right)-$ convex at $u \in X$ with respect to $H_{t}, p$, and $s$. Then $\left(\frac{f_{t}(\cdot)+\left(\cdot(\cdot)^{T} z_{t}\right.}{g_{t}(\cdot)-(\cdot)^{T} v_{t}}\right)$ is also higher-order $\left(\bar{C}, \bar{\alpha}_{t}, \bar{\gamma}_{t}, \bar{\rho}_{t}, \bar{d}_{t}\right)$-convex at $u \in X$ with respect to $\bar{H}_{t}, p$, and s, where

$$
\begin{aligned}
& \bar{\alpha}_{t}(x, u)=\left(\frac{g_{t}(u)-u^{T} v_{t}}{g_{t}(x)-x^{T} v_{t}}\right) \alpha_{t}(x, u), \quad \bar{\gamma}_{t}(x, u)=\gamma_{t}(x, u), \\
& \bar{\rho}_{t}=\rho_{t}\left(1+\frac{f_{t}(u)+u^{T} z_{t}}{g_{t}(u)-u^{T} v_{t}}\right), \quad \bar{H}_{t}(u, \cdot)=\left(\frac{1}{\left(g_{t}(u)-u^{T} v_{t}\right)}+\frac{f_{t}(u)+u^{T} z_{t}}{\left(g_{t}(u)-u^{T} v_{t}\right)^{2}}\right) H_{t}(u, \cdot), \\
& \bar{d}_{t}(x, u)=\left(\frac{d_{t}(x, u)}{g_{t}(x)-x^{T} v_{t}}\right),
\end{aligned}
$$

and

$$
\begin{aligned}
& \bar{C}_{x, u}(a)=\left(\frac{f_{t}(u)+u^{T} z_{t}+g_{t}(u)-u^{T} v_{t}}{\left(g_{t}(u)-u^{T} v_{t}\right)^{2}}\right) C_{x, u}\left(\frac{\left(g_{t}(u)-u^{T} v_{t}\right)^{2} a}{f_{t}(u)+u^{T} z_{t}+g_{t}(u)-u^{T} v_{t}}\right), \\
& a=\nabla\left(\frac{f_{t}(u)+u^{T} z_{t}}{g_{t}(u)-u^{T} v_{t}}\right)+\nabla_{p} \bar{H}_{t}(u, p) .
\end{aligned}
$$

Proof For any $x, u \in X$,

$$
\begin{aligned}
\left(\frac{f_{t}(x)+x^{T} z_{t}}{g_{t}(x)-x^{T} v_{t}}-\frac{f_{t}(u)+u^{T} z_{t}}{g_{t}(u)-u^{T} v_{t}}\right)= & \left(\frac{f_{t}(x)+x^{T} z_{t}-f_{t}(u)-u^{T} z_{t}}{g_{t}(x)-x^{T} v_{t}}\right) \\
& -\left[f_{t}(u)+u^{T} z_{t}\right]\left(\frac{g_{t}(x)-x^{T} v_{t}-g_{t}(u)+u^{T} v_{t}}{\left(g_{t}(x)-x^{T} v_{t}\right)\left(g_{t}(u)-u^{T} v_{t}\right)}\right) .
\end{aligned}
$$

Since $f_{t}(\cdot)+(\cdot)^{T} z_{t}$ and $-\left(g_{t}(\cdot)-(\cdot)^{T} v_{t}\right)$ are $\left(C, \alpha_{t}, \gamma_{t}, \rho_{t}, d_{t}\right)$-convex at $u \in X$ with respect to $H_{t}, p$, and $s$, we have

$$
\begin{aligned}
& \frac{1}{\alpha_{t}(x, u)}\left(\frac{f_{t}(x)+x^{T} z_{t}}{g_{t}(x)-x^{T} v_{t}}-\frac{f_{t}(u)+u^{T} z_{t}}{g_{t}(u)-u^{T} v_{t}}\right) \\
& \geq \frac{1}{\left(g_{t}(x)-x^{T} v_{t}\right)}\left\{C_{x, u}\left(\nabla\left(f_{t}(u)+u^{T} z_{t}\right)+\nabla_{p} H_{t}(u, p)\right)\right. \\
& \left.\quad+\frac{1}{\gamma_{t}(x, u)}\left[H_{t}(u, s)-s^{T} \nabla_{s} H_{t}(u, s)\right]+\frac{\rho_{t} d_{t}(x, u)}{\alpha_{t}(x, u)}\right\}
\end{aligned}
$$




$$
\begin{aligned}
& +\frac{f_{t}(u)+u^{T} z_{t}}{\left(g_{t}(x)-x^{T} v_{t}\right)\left(g_{t}(u)-u^{T} v_{t}\right)}\left\{C_{x, u}\left(-\nabla\left(g_{t}(u)-u^{T} v_{t}\right)+\nabla_{p} H_{t}(u, p)\right)\right. \\
& \left.+\frac{1}{\gamma_{t}(x, u)}\left[H_{t}(u, s)-s^{T} \nabla_{s} H_{t}(u, s)\right]+\frac{\rho_{t} d_{t}(x, u)}{\alpha_{t}(x, u)}\right\},
\end{aligned}
$$

which implies

$$
\begin{aligned}
& \frac{1}{\alpha_{t}(x, u)}\left(\frac{f_{t}(x)+x^{T} z_{t}}{g_{t}(x)-x^{T} v_{t}}-\frac{f_{t}(u)+u^{T} z_{t}}{g_{t}(u)-u^{T} v_{t}}\right) \\
& \geq\left(\frac{f_{t}(u)+u^{T} z_{t}+g_{t}(u)-u^{T} v_{t}}{\left(g_{t}(x)-x^{T} v_{t}\right)\left(g_{t}(u)-u^{T} v_{t}\right)}\right)\left(\frac{g_{t}(u)-u^{T} v_{t}}{f_{t}(u)+u^{T} z_{t}+g_{t}(u)-u^{T} v_{t}}\right) \\
& \quad \times\left\{C_{x, u}\left(\nabla\left(f_{t}(u)+u^{T} z_{t}\right)+\nabla_{p} H_{t}(u, p)\right)\right. \\
& \left.\quad+\frac{1}{\gamma_{t}(x, u)}\left[H_{t}(u, s)-s^{T} \nabla_{s} H_{t}(u, s)\right]+\frac{\rho_{t} d_{t}(x, u)}{\alpha_{t}(x, u)}\right\} \\
& \quad+\left(\frac{f_{t}(u)+u^{T} z_{t}+g_{t}(u)-u^{T} v_{t}}{\left(g_{t}(x)-x^{T} v_{t}\right)\left(g_{t}(u)-u^{T} v_{t}\right)}\right)\left(\frac{f_{t}(u)+u^{T} z_{t}}{f_{t}(u)+u^{T} z_{t}+g_{t}(u)-u^{T} v_{t}}\right) \\
& \quad \times\left\{C_{x, u}\left(-\nabla\left(g_{t}(u)-u^{T} v_{t}\right)+\nabla_{p} H_{t}(u, p)\right)\right. \\
& \left.\quad+\frac{1}{\gamma_{t}(x, u)}\left[H_{t}(u, s)-s^{T} \nabla_{s} H_{t}(u, s)\right]+\frac{\rho_{t} d_{t}(x, u)}{\alpha_{t}(x, u)}\right\} .
\end{aligned}
$$

This further yields

$$
\begin{aligned}
& \frac{1}{\alpha_{t}(x, u)}\left(\frac{f_{t}(x)+x^{T} z_{t}}{g_{t}(x)-x^{T} v_{t}}-\frac{f_{t}(u)+u^{T} z_{t}}{g_{t}(u)-u^{T} v_{t}}\right) \\
& \geq \\
& \quad\left(\frac{f_{t}(u)+u^{T} z_{t}+g_{t}(u)-u^{T} v_{t}}{\left(g_{t}(x)-x^{T} v_{t}\right)\left(g_{t}(u)-u^{T} v_{t}\right)}\right) \\
& \quad \times\left\{C _ { x , u } \left(\frac { ( g _ { t } ( u ) - u ^ { T } v _ { t } ) ^ { 2 } } { f _ { t } ( u ) + u ^ { T } z _ { t } + g _ { t } ( u ) - u ^ { T } v _ { t } } \left(\nabla\left(\frac{f_{t}(u)+u^{T} z_{t}}{g_{t}(u)-u^{T} v_{t}}\right)\right.\right.\right. \\
& \left.\left.\left.\quad+\left(\frac{1}{g_{t}(u)-u^{T} v_{t}}+\frac{f_{t}(u)+u^{T} z_{t}}{\left(g_{t}(u)-u^{T} v_{t}\right)^{2}}\right) \nabla_{p} H_{t}(u, p)\right)\right)\right\}+\frac{1}{\gamma_{t}(x, u)\left(g_{t}(x)-x^{T} v_{t}\right)} \\
& \quad \times\left[\left(1+\frac{f_{t}(u)+u^{T} z_{t}}{\left(g_{t}(u)-u^{T} v_{t}\right)}\right)\left(H_{t}(u, s)-s^{T} \nabla_{s} H_{t}(u, s)\right)\right] \\
& \quad+\frac{\rho_{t} d_{t}(x, u)}{\alpha_{t}(x, u)\left(g_{t}(x)-x^{T} v_{t}\right)}\left(1+\frac{f_{t}(u)+u^{T} z_{t}}{\left(g_{t}(u)-u^{T} v_{t}\right)}\right) .
\end{aligned}
$$

Multiplying by $\left(\frac{g_{t}(x)-x^{T} v_{t}}{g_{t}(u)-u^{T} v_{t}}\right)$, the above inequality gives

$$
\begin{aligned}
& \frac{\left(g_{t}(x)-x^{T} v_{t}\right)}{\alpha_{t}(x, u)\left(g_{t}(u)-u^{T} v_{t}\right)}\left(\frac{f_{t}(x)+x^{T} z_{t}}{g_{t}(x)-x^{T} v_{t}}-\frac{f_{t}(u)+u^{T} z_{t}}{g_{t}(u)-u^{T} v_{t}}\right) \\
& \geq\left(\frac{f_{t}(u)+u^{T} z_{t}+g_{t}(u)-u^{T} v_{t}}{\left(g_{t}(u)-u^{T} v_{t}\right)^{2}}\right) \\
& \quad \times\left\{C _ { x , u } \left(\frac { ( g _ { t } ( u ) - u ^ { T } v _ { t } ) ^ { 2 } } { f _ { t } ( u ) + u ^ { T } z _ { t } + g _ { t } ( u ) - u ^ { T } v _ { t } } \left(\nabla\left(\frac{f_{t}(u)+u^{T} z_{t}}{g_{t}(u)-u^{T} v_{t}}\right)\right.\right.\right.
\end{aligned}
$$




$$
\begin{aligned}
& \left.\left.\left.+\left(\frac{1}{g_{t}(u)-u^{T} v_{t}}+\frac{f_{t}(u)+u^{T} z_{t}}{\left(g_{t}(u)-u^{T} v_{t}\right)^{2}}\right) \nabla_{p} H_{t}(u, p)\right)\right)\right\} \\
& +\frac{1}{\gamma_{t}(x, u)\left(g_{t}(u)-u^{T} v_{t}\right)}\left[\left(1+\frac{f_{t}(u)+u^{T} z_{t}}{\left(g_{t}(u)-u^{T} v_{t}\right)}\right)\left(H_{t}(u, s)-s^{T} \nabla_{s} H_{t}(u, s)\right)\right] \\
& +\frac{\rho_{t} d_{t}(x, u)}{\alpha_{t}(x, u)\left(g_{t}(u)-u^{T} v_{t}\right)}\left(1+\frac{f_{t}(u)+u^{T} z_{t}}{\left(g_{t}(u)-u^{T} v_{t}\right)}\right) .
\end{aligned}
$$

Setting

$$
\begin{aligned}
& \bar{\alpha}_{t}(x, u)=\left(\frac{g_{t}(u)-u^{T} v_{t}}{g_{t}(x)-x^{T} v_{t}}\right) \alpha_{t}(x, u), \quad \bar{\gamma}_{t}(x, u)=\gamma_{t}(x, u), \\
& \bar{\rho}_{t}=\rho_{t}\left(1+\frac{f_{t}(u)+u^{T} z_{t}}{g_{t}(u)-u^{T} v_{t}}\right), \\
& \bar{H}_{t}(u, \cdot)=\left(\frac{1}{\left(g_{t}(u)-u^{T} v_{t}\right)}+\frac{f_{t}(u)+u^{T} z_{t}}{\left(g_{t}(u)-u^{T} v_{t}\right)^{2}}\right) H_{t}(u, \cdot), \quad \bar{d}_{t}(x, u)=\left(\frac{d_{t}(x, u)}{g_{t}(x)-x^{T} v_{t}}\right), \\
& \bar{C}_{(x, u)}(a)=\left(\frac{f_{t}(u)+u^{T} z_{t}+g_{t}(u)-u^{T} v_{t}}{\left(g_{t}(u)-u^{T} v_{t}\right)^{2}}\right) C_{x, u}\left(\frac{\left(g_{t}(u)-u^{T} v_{t}\right)^{2} a}{f_{t}(u)+u^{T} z_{t}+g_{t}(u)-u^{T} v_{t}}\right),
\end{aligned}
$$

and

$$
a=\nabla\left(\frac{f_{t}(u)+u^{T} z_{t}}{g_{t}(u)-u^{T} v_{t}}\right)+\nabla_{p} \bar{H}_{t}(u, p)
$$

It follows that

$$
\begin{aligned}
& \frac{1}{\bar{\alpha}_{t}(x, u)}\left(\frac{f_{t}(x)+x^{T} z_{t}}{g_{t}(x)-x^{T} v_{t}}-\frac{f_{t}(u)+u^{T} z_{t}}{g_{t}(u)-u^{T} v_{t}}\right) \\
& \geq\left\{\bar{C}_{(x, u)}\left(\nabla\left(\frac{f_{t}(u)+u^{T} z_{t}}{g_{t}(u)-u^{T} v_{t}}\right)+\nabla_{p} \bar{H}_{t}(u, p)\right)\right. \\
& \left.\quad+\frac{1}{\bar{\gamma}_{t}(x, u)}\left[\bar{H}_{t}(u, s)-s^{T} \nabla_{s} \bar{H}_{t}(u, s)\right]+\frac{\bar{\rho}_{t} \bar{d}_{t}(x, u)}{\bar{\alpha}_{t}(x, u)}\right\} .
\end{aligned}
$$

Hence, $\left(\frac{f_{t}(\cdot)+(\cdot)^{T} z_{t}}{g_{t}(\cdot)-(\cdot)^{T} z_{t}}\right)$ is higher-order $\left(\bar{C}, \bar{\alpha}_{t}, \bar{\gamma}_{t}, \bar{\rho}_{t}, \bar{d}_{t}\right)$-convex at $u \in X$ with respect to $\bar{H}_{t}, p$, and $s$.

Theorem 3.2 Let $X \subset R^{n}$ be an open convex set. Let $\psi_{i}: X \rightarrow R$ be higher-order $\left(C, \alpha_{i}, \gamma_{i}, \rho_{i}, d_{i}\right)$-convex at one point in $X$ with respect to $\phi_{i}, \rho_{i} \geq 0$, and for all $x \in X$, $\phi_{i}(x, \cdot)=-(\cdot)^{T} \nabla \psi_{i}(x), i=1,2, \ldots, k$; then only one of the following two cases holds:

(i) there exists $x \in X$ such that $\psi_{i}(x)<0, i=1,2, \ldots, k$;

(ii) there exists $\lambda \in R_{+}^{k} \backslash\{0\}$ such that $\sum_{i=1}^{k} \lambda_{i} \psi_{i}(x) \geq 0$, for all $x \in X$.

Proof If (i) has a solution, that is, there exists $x \in X$ such that $\psi_{i}(x)<0, i=1,2, \ldots, k$, then for every $\lambda \in R_{+}^{k} \backslash\{0\}$, we have $\sum_{i=1}^{k} \lambda_{i} \psi_{i}(x)<0$, which implies that (ii) does not hold.

If (i) has no solution, let $K=\psi(X)+\operatorname{int}\left(R_{+}^{k}\right), \psi=\left(\psi_{1}, \psi_{2}, \ldots, \psi_{k}\right)^{T}$, then $K \cap\left(-R_{+}^{k}\right)=\phi$. For any $z^{1}, z^{2} \in K$ and $\beta \in(0,1)$, there exist $x^{1}, x^{2} \in X$ and $s^{1}, s^{2} \in \operatorname{int}\left(R_{+}^{k}\right)$ such that

$$
\beta z^{1}+(1-\beta) z^{2}=\beta \psi\left(x^{1}\right)+(1-\beta) \psi\left(x^{2}\right)+\beta s^{1}+(1-\beta) s^{2} .
$$


Since $u=\beta x^{1}+(1-\beta) x^{2} \in X$ and $\phi_{i}(x, \cdot)=-(\cdot)^{T} \nabla \psi_{i}(x)$, it follows from the higher-order $\left(C, \alpha_{i}, \gamma_{i}, \rho_{i}, d_{i}\right)$-convexity of $\psi_{i}$ with respect to $\phi_{i}, p$, and $s$ that we have

$$
\begin{aligned}
& \frac{1}{\alpha_{i}\left(x^{j}, u\right)}\left[\psi_{i}\left(x^{j}\right)-\psi_{i}(u)\right] \\
& \quad \geq C_{x, u}\left[\nabla \psi_{i}(u)+\nabla_{p} \phi_{i}(u, p)\right]+\frac{1}{\gamma_{i}\left(x^{j}, u\right)}\left\{\phi_{i}(u, s)-s^{T}\left[\nabla_{s} \phi_{i}(u, s)\right]\right\}+\frac{\rho_{i} d\left(x^{j}, u\right)}{\alpha_{i}\left(x^{j}, u\right)} \\
& \quad=\frac{1}{\alpha_{i}\left(x^{j}, u\right)} \rho_{i} d\left(x^{j}, u\right), \quad j=1,2 .
\end{aligned}
$$

Thus, there exists $t \geq 0$ such that

$$
\beta \psi_{i}\left(x^{1}\right)+(1-\beta) \psi_{i}\left(x^{2}\right)=\psi_{i}\left(\beta x^{1}+(1-\beta) x^{2}\right)+t
$$

which implies that

$$
\beta z^{1}+(1-\beta) z^{2}=\beta \psi\left(x^{1}\right)+(1-\beta) \psi\left(x^{2}\right)+\beta s^{1}+(1-\beta) s^{2}+(t, t, \ldots, t)^{T}
$$

Since $R_{+}^{k}$ is a closed convex cone, we have $\beta z_{1}+(1-\beta) z_{2} \in K$, that is, $K$ is an open convex set. It follows from the convex separated theorem that there exists $\lambda \in R_{+}^{k} \backslash\{0\}$ such that, for all $x \in X, \sum_{i=1}^{k} \lambda_{i} \psi_{i}(x) \geq 0$.

We now discuss some optimality conditions for the problem (MFP).

Theorem 3.3 If $u \in X$ is a weakly efficient solution of (MFP), $f_{i}(\cdot)+(\cdot)^{T} z_{i}$, and $-\left(g_{i}(\cdot)-\right.$ $\left.(\cdot)^{T} v_{i}\right)$ are higher-order $\left(C, \alpha_{i}, \gamma_{i}, \rho_{i}, d_{i}\right)$-convex functions at $u \in X$ with respect to $\phi_{i}(u, \cdot)=$ $-\frac{\left(g_{i}(u)-u^{T} v_{i}\right)^{2}}{\left(f_{i}(u)+u^{T} z_{i}+g_{i}(u)-u^{T} v_{i}\right)} \times(\cdot)^{T} \nabla\left(\frac{f_{i}(u)+u^{T} z_{i}}{g_{i}(u)-u^{T} v_{i}}\right)$, and $\rho_{i} \geq 0, i=1,2, \ldots, k$. Then there exists $0 \leq \bar{\lambda} \in$ $R^{k}, \bar{\lambda} \neq 0$ such that $u$ is an optimal solution of $\left(\mathrm{FP}_{\bar{\lambda}}\right)$.

Proof If $u \in X$ is a weakly efficient solution of (MFP), then there does not exist any $x \in X$ such that

$$
\psi_{j}(x)=\left(\frac{f_{j}(x)+x^{T} z_{j}}{g_{j}(x)-x^{T} v_{j}}-\frac{f_{j}(u)+u^{T} z_{j}}{g_{j}(u)-u^{T} v_{j}}\right)<0, \quad j=1,2, \ldots, k .
$$

Since $f_{j}(\cdot)+(\cdot)^{T} z_{j}$ and $-\left(g_{j}(\cdot)-(\cdot)^{T} v_{j}\right)$ are higher-order $\left(C, \alpha_{j}, \gamma_{j}, \rho_{j}, d_{j}\right)$-convex at $u$ with respect to $\phi_{j}, p$ and $s, j=1,2, \ldots, k$, it follows from Theorem 3.1 that $\psi_{j}(x)=\left(\frac{f_{j}(x)+x^{T} z_{j}}{g_{j}(x)-x^{T} v_{j}}-\right.$ $\left.\frac{f_{j}(u)+u^{T} z_{j}}{g_{j}(u)-u^{T} v_{j}}\right)$ is higher-order $\left(\bar{C}, \bar{\alpha}_{j}, \bar{\gamma}_{j}, \bar{\rho}_{j}, \bar{d}_{j}\right)$-convex at $u$ with respect to $\bar{\phi}_{j}, p$, and $s, j=$ $1,2, \ldots, k$, where

$$
\begin{aligned}
& \bar{\alpha}_{j}(x, u)=\left(\frac{g_{j}(x)-x^{T} v_{j}}{g_{j}(u)-u^{T} v_{j}}\right) \alpha_{j}(x, u), \quad \bar{\gamma}_{j}(x, u)=\gamma_{j}(x, u), \\
& \bar{\rho}_{j}=\rho_{j}\left(1+\frac{f_{j}(u)+u^{T} z_{j}}{g_{j}(u)-u^{T} v_{j}}\right), \\
& \bar{\phi}_{j}(u, \cdot)=\left(\frac{1}{g_{j}(u)-u^{T} v_{j}}+\frac{f_{j}(u)+u^{T} z_{j}}{\left(g_{j}(u)-u^{T} v_{j}\right)^{2}}\right) \phi_{j}(u, \cdot)=-(\cdot)^{T} \nabla\left(\frac{f_{j}(u)+u^{T} z_{j}}{g_{j}(u)-u^{T} v_{j}}\right) \\
&=-(\cdot)^{T} \nabla \psi_{j}(u),
\end{aligned}
$$




$$
\bar{d}_{j}(x, u)=\left(\frac{d_{j}(x, u)}{g_{j}(x)-x^{T} v_{j}}\right)
$$

and

$$
\begin{aligned}
& \bar{C}_{x, u}(a)=\frac{f_{j}(u)+u^{T} z_{j}+g_{j}(u)-u^{T} v_{j}}{\left(g_{j}(u)-u^{T} v_{j}\right)^{2}} C_{x, u}\left(\frac{\left(g_{j}(u)-u^{T} v_{j}\right)^{2} a}{f_{j}(u)+u^{T} z_{j}+g_{j}(u)-u^{T} v_{j}}\right), \\
& a=\nabla\left(\frac{f_{j}(u)+u^{T} z_{j}}{g_{j}(u)-u^{T} v_{j}}\right)+\nabla_{p} \bar{\phi}_{j}(u, p) .
\end{aligned}
$$

Using Theorem 3.2, there exists $0 \leq \bar{\lambda} \in R^{k}, \bar{\lambda} \neq 0$ such that $\sum_{j=1}^{k} \bar{\lambda}_{j} \psi_{j}(x) \geq 0$, that is,

$$
\sum_{j=1}^{k} \bar{\lambda}_{j}\left(\frac{f_{j}(x)+x^{T} z_{j}}{g_{j}(x)-x^{T} v_{j}}\right) \geq \sum_{j=1}^{k} \bar{\lambda}_{j}\left(\frac{f_{j}(u)+u^{T} z_{j}}{g_{j}(u)-u^{T} v_{j}}\right)
$$

which implies that $u$ is an optimal solution of $\left(\mathrm{FP}_{\bar{\lambda}}\right)$.

Theorem 3.4 (Necessary condition) [18] Assume that $\bar{x}$ is an efficient solution of (MFP) and the Slater constraint qualification is satisfied on $X$. Then there exist $\bar{\lambda} \in R^{k}, \bar{\mu} \in R^{m}$, $\bar{z}_{i} \in R^{n}, \bar{v}_{i} \in R^{n}$, and $\bar{w}_{j} \in R^{n}, i=1,2, \ldots, k, j=1,2, \ldots, m$, such that

$$
\begin{aligned}
& \sum_{i=1}^{k} \bar{\lambda}_{i} \nabla\left(\frac{f_{i}(\bar{x})+\bar{x}^{T} \bar{z}_{i}}{g_{i}(\bar{x})-\bar{x}^{T} \bar{v}_{i}}\right)+\sum_{j=1}^{m} \bar{\mu}_{j} \nabla\left(h_{j}(\bar{x})+\bar{x}^{T} \bar{w}_{j}\right)=0, \\
& \sum_{j=1}^{m} \bar{\mu}_{j}\left(h_{j}(\bar{x})+\bar{x}^{T} \bar{w}_{j}\right)=0, \\
& \bar{x}^{T} \bar{z}_{i}=S\left(\bar{x} \mid C_{i}\right), \quad i=1,2, \ldots, k \\
& \bar{x}^{T} \bar{v}_{i}=S\left(\bar{x} \mid D_{i}\right), \quad i=1,2, \ldots, k, \\
& \bar{x}^{T} \bar{w}_{j}=S\left(\bar{x} \mid E_{j}\right), \quad j=1,2, \ldots, m, \\
& \bar{z}_{i} \in C_{i}, \quad \bar{v}_{i} \in D_{i}, \quad \bar{w}_{j} \in E_{j}, \quad i=1,2, \ldots, k, j=1,2, \ldots, m, \\
& \bar{\lambda}_{i}>0, \quad \bar{\mu}_{j} \geq 0, \quad i=1,2, \ldots, k, j=1,2, \ldots, m .
\end{aligned}
$$

Theorem 3.5 (Sufficient condition) Let $u$ be a feasible solution of (MFP). Assume that there exist $\lambda_{i}>0, i=1,2, \ldots, k$ and $\mu_{j} \geq 0, j=1,2, \ldots, m$, such that

$$
\begin{aligned}
& \sum_{i=1}^{k} \lambda_{i} \nabla\left(\frac{f_{i}(u)+u^{T} z_{i}}{g_{i}(u)-u^{T} v_{i}}\right)+\sum_{j=1}^{m} \mu_{j} \nabla\left(h_{j}(u)+u^{T} w_{j}\right)=0, \\
& \sum_{j=1}^{m} \mu_{j}\left(h_{j}(u)+u^{T} w_{j}\right)=0, \\
& u^{T} z_{i}=S\left(u \mid C_{i}\right), \quad i=1,2, \ldots, k, \\
& u^{T} v_{i}=S\left(u \mid D_{i}\right), \quad i=1,2, \ldots, k, \\
& u^{T} w_{j}=S\left(u \mid E_{j}\right), \quad j=1,2, \ldots, m,
\end{aligned}
$$


$z_{i} \in C_{i}, \quad v_{i} \in D_{i}, \quad w_{j} \in E_{j}, \quad i=1,2, \ldots, k, j=1,2, \ldots, m$

Let for any $i=1,2, \ldots, k, j=1,2, \ldots, m$,

(i) $\left(f_{i}(\cdot)+(\cdot)^{T} z_{i}\right)$ and $-\left(g_{i}(\cdot)-(\cdot)^{T} v_{i}\right)$ be higher-order $\left(C, \alpha_{i}, \gamma_{i}, \rho_{i}, d_{i}\right)$-convex at $u$, with respect to $H_{i}, p$, and $s$,

(ii) $\left(h_{j}(\cdot)+(\cdot)^{T} w_{j}\right)$ be higher-order $\left(C, \xi_{j}, \delta_{j}, \eta_{j}, c_{j}\right)$-convex at $u$, with respect to $K_{j}, q$, and $r$,

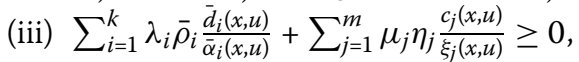

(iv) $\bar{\gamma}_{i}(x, u)=\zeta(x, u), \xi_{j}(x, u)=\sigma(x, u)$, and $\delta_{j}(x, u)=\sigma^{\prime}(x, u)$,

(v) $\sum_{i=1}^{k} \lambda_{i}\left(\nabla_{p} \bar{H}_{i}(u, p)\right)+\sum_{j=1}^{m} \mu_{j}\left(\nabla_{q} K_{j}(u, q)\right)=0, \sum_{i=1}^{k} \lambda_{i}\left(\bar{H}_{i}(u, s)-s^{T} \nabla_{s} \bar{H}_{i}(u, s)\right) \geq 0$ and $\sum_{j=1}^{m} \mu_{j}\left(K_{j}(u, r)-r^{T} \nabla_{r} K_{j}(u, r)\right) \geq 0$,

where

$$
\begin{array}{ll}
\bar{\alpha}_{i}(x, u)=\left(\frac{g_{i}(u)-u^{T} v_{i}}{g_{i}(x)-x^{T} v_{i}}\right) \alpha_{i}(x, u), \bar{\gamma}_{i}(x, u)=\gamma_{i}(x, u), & \bar{\rho}_{i}=\rho_{i}\left(1+\frac{f_{i}(u)+u^{T} z_{i}}{g_{i}(u)-u^{T} v_{i}}\right), \\
\bar{H}_{i}(u, \cdot)=\left(\frac{1}{g_{i}(u)-u^{T} v_{i}}+\frac{f_{i}(u)+u^{T} z_{i}}{\left(g_{i}(u)-u^{T} v_{i}\right)^{2}}\right) H_{i}(u, \cdot), & \bar{d}_{i}(x, u)=\left(\frac{d_{i}(x, u)}{g_{i}(x)-x^{T} v_{i}}\right) .
\end{array}
$$

Then $u$ is an efficient solution of (MFP).

Proof Suppose $u$ is not an efficient solution of (MFP), then there exists $x \in X^{0}$ such that

$$
\frac{f_{i}(x)+S\left(x \mid C_{i}\right)}{g_{i}(x)-S\left(x \mid D_{i}\right)} \leq \frac{f_{i}(u)+S\left(u \mid C_{i}\right)}{g_{i}(u)-S\left(u \mid D_{i}\right)}, \quad \text { for all } i=1,2, \ldots, k
$$

and

$$
\frac{f_{r}(x)+S\left(x \mid C_{r}\right)}{g_{r}(x)-S\left(x \mid D_{r}\right)}<\frac{f_{r}(u)+S\left(u \mid C_{r}\right)}{g_{r}(u)-S\left(u \mid D_{r}\right)}, \quad \text { for some } r=1,2, \ldots, k
$$

which implies

$$
\begin{aligned}
& \frac{f_{i}(x)+x^{T} z_{i}}{g_{i}(x)-x^{T} v_{i}} \leq \frac{f_{i}(x)+S\left(x \mid C_{i}\right)}{g_{i}(x)-S\left(x \mid D_{i}\right)} \leq \frac{f_{i}(u)+S\left(u \mid C_{i}\right)}{g_{i}(u)-S\left(u \mid D_{i}\right)}=\frac{f_{i}(u)+u^{T} z_{i}}{g_{i}(u)-u^{T} v_{i}}, \\
& \quad \text { for all } i=1,2, \ldots, k
\end{aligned}
$$

and

$$
\begin{aligned}
& \frac{f_{r}(x)+x^{T} z_{r}}{g_{r}(x)-x^{T} v_{r}} \leq \frac{f_{r}(x)+S\left(x \mid C_{r}\right)}{g_{r}(x)-S\left(x \mid D_{r}\right)}<\frac{f_{r}(u)+S\left(u \mid C_{r}\right)}{g_{r}(u)-S\left(u \mid D_{r}\right)}=\frac{f_{r}(u)+u^{T} z_{r}}{g_{r}(u)-u^{T} v_{r}} \\
& \quad \text { for some } r=1,2, \ldots, k .
\end{aligned}
$$

Since $\frac{\lambda_{i}}{\bar{\alpha}_{i}(x, u)}>0, i=1,2, \ldots, k$, the inequalities (1) and (2) give

$$
\sum_{i=1}^{k} \frac{\lambda_{i}}{\bar{\alpha}_{i}(x, u)}\left(\frac{f_{i}(x)+x^{T} z_{i}}{g_{i}(x)-x^{T} \nu_{i}}-\frac{f_{i}(u)+u^{T} z_{i}}{g_{i}(u)-u^{T} \nu_{i}}\right)<0 .
$$


From Theorem 3.1, for each $i, 1 \leq i \leq k,\left(\frac{f_{i}(\cdot)+(\cdot)^{T} z_{i}}{g_{i}(\cdot)-(\cdot)^{T} v_{i}}\right)$ is higher-order $\left(\bar{C}, \bar{\alpha}_{i}, \bar{\gamma}_{i}, \bar{\rho}_{i}, \bar{d}_{i}\right)$-convex at $u \in X^{0}$ with respect to $\bar{H}_{i}, p$, and $s$, therefore

$$
\begin{aligned}
& \frac{1}{\bar{\alpha}_{i}(x, u)}\left(\frac{f_{i}(x)+x^{T} z_{i}}{g_{i}(x)-x^{T} v_{i}}-\frac{f_{i}(u)+u^{T} z_{i}}{g_{i}(u)-u^{T} v_{i}}\right) \\
& \geq \bar{C}_{x, u}\left(\nabla\left(\frac{f_{i}(u)+u^{T} z_{i}}{g_{i}(u)-u^{T} v_{i}}\right)+\nabla_{p} \bar{H}_{i}(u, p)\right) \\
& \quad+\frac{1}{\bar{\gamma}_{i}(x, u)}\left[\bar{H}_{i}(u, s)-s^{T} \nabla_{s} \bar{H}_{i}(u, s)\right]+\frac{\bar{\rho}_{i} \bar{d}_{i}(x, u)}{\bar{\alpha}_{i}(x, u)},
\end{aligned}
$$

where

$$
\begin{aligned}
& \bar{\alpha}_{i}(x, u)=\left(\frac{g_{i}(u)-u^{T} v_{i}}{g_{i}(x)-x^{T} v_{i}}\right) \alpha_{i}(x, u), \quad \bar{\gamma}_{i}(x, u)=\gamma_{i}(x, u), \\
& \bar{\rho}_{i}=\rho_{i}\left(1+\frac{f_{i}(u)+u^{T} z_{i}}{g_{i}(u)-u^{T} v_{i}}\right), \\
& \bar{H}_{i}(u, \cdot)=\left(\frac{1}{g_{i}(u)-u^{T} v_{i}}+\frac{f_{i}(u)+u^{T} z_{i}}{\left(g_{i}(u)-u^{T} v_{i}\right)^{2}}\right) H_{i}(u, \cdot), \quad \bar{d}_{i}(x, u)=\left(\frac{d_{i}(x, u)}{g_{i}(x)-x^{T} v_{i}}\right),
\end{aligned}
$$

and

$$
\begin{aligned}
& \bar{C}_{x, u}(a)=\left(\frac{f_{i}(u)+u^{T} z_{i}+g_{i}(u)-u^{T} v_{i}}{\left(g_{i}(u)-u^{T} v_{i}\right)^{2}}\right) C_{x, u}\left(\frac{\left(g_{i}(u)-u^{T} v_{i}\right)^{2} a}{f_{i}(u)+u^{T} z_{i}+g_{i}(u)-u^{T} v_{i}}\right), \\
& a=\nabla\left(\frac{f_{i}(u)+u^{T} z_{i}}{g_{i}(u)-u^{T} v_{i}}\right)+\nabla_{p} \bar{H}_{i}(u, p) .
\end{aligned}
$$

Also, by the higher-order $\left(C, \xi_{j}, \delta_{j}, \eta_{j}, c_{j}\right)$-convexity of $\left(h_{j}(\cdot)+(\cdot)^{T} w_{j}\right)$ at $u$ with respect to $K_{j}$, $q$, and $r, j=1,2, \ldots, m$, we have

$$
\begin{aligned}
& \frac{1}{\xi_{j}(x, u)}\left[h_{j}(x)+x^{T} w_{j}-h_{j}(u)-u^{T} w_{j}\right] \\
& \quad \geq C_{x, u}\left(\nabla\left(h_{j}(u)+u^{T} w_{j}\right)+\nabla_{q} K_{j}(u, q)\right) \\
& \quad+\frac{1}{\delta_{j}(x, u)}\left[K_{j}(u, r)-r^{T} \nabla_{r} K_{j}(u, r)\right]+\frac{\eta_{j} c_{j}(x, u)}{\xi_{j}(x, u)} .
\end{aligned}
$$

Let $\tau=\sum_{i=1}^{k} \lambda_{i} \frac{\left(f_{i}(u)+u^{T} z_{i}+g_{i}(u)-u^{T} v_{i}\right)}{\left(g_{i}(u)-u^{T} v_{i}\right)^{2}}+\sum_{j=1}^{m} \mu_{j}>0$.

Adding the inequalities obtained by multiplying (4) by $\frac{\lambda_{i}}{\tau}$ and (5) by $\frac{\mu_{j}}{\tau}$, we get

$$
\begin{aligned}
& \sum_{i=1}^{k} \frac{\lambda_{i}}{\tau \bar{\alpha}_{i}(x, u)}\left(\frac{f_{i}(x)+x^{T} z_{i}}{g_{i}(x)-x^{T} v_{i}}-\frac{f_{i}(u)+u^{T} z_{i}}{g_{i}(u)-u^{T} v_{i}}\right) \\
& \quad+\sum_{j=1}^{m} \frac{\mu_{j}}{\tau \xi_{j}(x, u)}\left[h_{j}(x)+x^{T} w_{j}-h_{j}(u)-u^{T} w_{j}\right] \\
& \geq \sum_{i=1}^{k} \frac{\lambda_{i}}{\tau} \frac{\left(f_{i}(u)+u^{T} z_{i}+g_{i}(u)-u^{T} v_{i}\right)}{\left(g_{i}(u)-u^{T} v_{i}\right)^{2}} C_{x, u}\left[\left(\frac{\left(g_{i}(u)-u^{T} v_{i}\right)^{2}}{f_{i}(u)+u^{T} z_{i}+g_{i}(u)-u^{T} v_{i}}\right)\right.
\end{aligned}
$$




$$
\begin{aligned}
& \times\left(\nabla\left(\frac{f_{i}(u)+u^{T} z_{i}}{g_{i}(u)-u^{T} v_{i}}\right)+\left(\frac{1}{g_{i}(u)-u^{T} v_{i}}+\frac{f_{i}(u)+u^{T} z_{i}}{\left(g_{i}(u)-u^{T} v_{i}\right)^{2}}\right) \nabla_{p} H_{i}(u, p)\right) \\
& \left.+\sum_{i=1}^{k} \frac{\lambda_{i}}{\tau} \frac{1}{\bar{\gamma}_{i}(x, u)}\left(\frac{1}{g_{i}(u)-u^{T} v_{i}}+\frac{f_{i}(u)+u^{T} z_{i}}{\left(g_{i}(u)-u^{T} v_{i}\right)^{2}}\right)\left(H_{i}(u, s)-s^{T} \nabla_{s} H_{i}(u, s)\right)\right] \\
& +\sum_{i=1}^{k} \frac{\lambda_{i}}{\tau} \frac{\rho_{i} d_{i}(x, u)}{\alpha_{i}(x, u)}\left(\frac{1}{g_{i}(u)-u^{T} v_{i}}+\frac{f_{i}(u)+u^{T} z_{i}}{\left(g_{i}(u)-u^{T} v_{i}\right)^{2}}\right) \\
& +\sum_{j=1}^{m} \frac{\mu_{j}}{\tau} C_{x, u}\left(\nabla\left(h_{j}(u)+u^{T} w_{j}\right)+\nabla_{q} K_{j}(u, q)\right) \\
& +\sum_{j=1}^{m} \frac{\mu_{j}}{\tau} \frac{1}{\delta_{j}(x, u)}\left[K_{j}(u, r)-r^{T} \nabla_{r} K_{j}(u, r)\right]+\sum_{j=1}^{m} \frac{\mu_{j}}{\tau} \frac{\eta_{j} c_{j}(x, u)}{\xi_{j}(x, u)} .
\end{aligned}
$$

Further, using the convexity of $C$, we have

$$
\begin{aligned}
\sum_{i=1}^{k} & \frac{\lambda_{i}}{\tau \bar{\alpha}_{i}(x, u)}\left(\frac{f_{i}(x)+x^{T} z_{i}}{g_{i}(x)-x^{T} v_{i}}-\frac{f_{i}(u)+u^{T} z_{i}}{g_{i}(u)-u^{T} v_{i}}\right) \\
& +\sum_{j=1}^{m} \frac{\mu_{j}}{\tau \xi_{j}(x, u)}\left[h_{j}(x)+x^{T} w_{j}-h_{j}(u)-u^{T} w_{j}\right] \\
\geq & C_{x, u}\left(\frac { 1 } { \tau } \left(\sum_{i=1}^{k} \lambda_{i} \nabla\left(\frac{f_{i}(u)+u^{T} z_{i}}{g_{i}(u)-u^{T} v_{i}}\right)+\sum_{i=1}^{k} \lambda_{i} \nabla_{p} \bar{H}_{i}(u, p)\right.\right. \\
& \left.\left.+\sum_{j=1}^{m} \mu_{j} \nabla\left(h_{j}(u)+u^{T} w_{j}\right)+\sum_{j=1}^{m} \mu_{j} \nabla_{q} K_{j}(u, q)\right)\right) \\
& +\sum_{i=1}^{k} \frac{\lambda_{i}}{\tau} \frac{1}{\bar{\gamma}_{i}(x, u)}\left(\bar{H}_{i}(u, s)-s^{T} \nabla_{s} \bar{H}_{i}(u, s)\right)+\sum_{i=1}^{k} \frac{\lambda_{i}}{\tau} \frac{\bar{\rho}_{i} \bar{d}_{i}(x, u)}{\bar{\alpha}_{i}(x, u)} \\
& +\sum_{j=1}^{m} \frac{\mu_{j}}{\tau} \frac{1}{\delta_{j}(x, u)}\left[K_{j}(u, r)-r^{T} \nabla_{r} K_{j}(u, r)\right]+\sum_{j=1}^{m} \frac{\mu_{j}}{\tau} \frac{\eta_{j} c_{j}(x, u)}{\xi_{j}(x, u)} .
\end{aligned}
$$

It follows from hypotheses (iii)-(v) that

$$
\begin{aligned}
& \sum_{i=1}^{k} \frac{\lambda_{i}}{\tau \bar{\alpha}_{i}(x, u)}\left(\frac{f_{i}(x)+x^{T} z_{i}}{g_{i}(x)-x^{T} v_{i}}-\frac{f_{i}(u)+u^{T} z_{i}}{g_{i}(u)-u^{T} v_{i}}\right) \\
& \quad+\sum_{j=1}^{m} \frac{\mu_{j}}{\tau \sigma(x, u)}\left[h_{j}(x)+x^{T} w_{j}-h_{j}(u)-u^{T} w_{j}\right] \\
& \geq C_{x, u}\left(\frac{1}{\tau}\left(\sum_{i=1}^{k} \lambda_{i} \nabla\left(\frac{f_{i}(u)+u^{T} z_{i}}{g_{i}(u)-u^{T} v_{i}}\right)+\sum_{j=1}^{m} \mu_{j} \nabla\left(h_{j}(u)+u^{T} w_{j}\right)\right)\right) .
\end{aligned}
$$

Using the fact $C_{x, u}(0)=0$ and $\sum_{j=1}^{m} \mu_{j}\left(h_{j}(u)+u^{T} w_{j}\right)=0$, we get

$$
\sum_{i=1}^{k} \frac{\lambda_{i}}{\tau \bar{\alpha}_{i}(x, u)}\left(\frac{f_{i}(x)+x^{T} z_{i}}{g_{i}(x)-x^{T} v_{i}}-\frac{f_{i}(u)+u^{T} z_{i}}{g_{i}(u)-u^{T} v_{i}}\right)+\sum_{i=1}^{m} \frac{\mu_{j}}{\tau \sigma(x, u)}\left[h_{j}(x)+x^{T} w_{j}\right] \geq 0 .
$$


Finally, using feasibility of the primal problem (MFP), we have

$$
\sum_{i=1}^{k} \frac{\lambda_{i}}{\bar{\alpha}_{i}(x, u)}\left(\frac{f_{i}(x)+x^{T} z_{i}}{g_{i}(x)-x^{T} v_{i}}-\frac{f_{i}(u)+u^{T} z_{i}}{g_{i}(u)-u^{T} v_{i}}\right) \geq 0,
$$

which contradicts (3). Therefore, $u$ is an efficient solution of (MFP).

\section{Duality model}

Consider the following higher-order Mond-Weir type dual (MFD) of (MFP):

$$
\begin{aligned}
& \text { (MFD) } \begin{array}{r}
\text { maximize } G(u)=\left\{\frac{f_{1}(u)+u^{T} z_{1}}{g_{1}(u)-u^{T} v_{1}}, \frac{f_{2}(u)+u^{T} z_{2}}{g_{2}(u)-u^{T} v_{2}}, \ldots, \frac{f_{k}(u)+u^{T} z_{k}}{g_{k}(u)-u^{T} v_{k}}\right\} \\
\text { subject to } \\
\quad \sum_{i=1}^{k} \lambda_{i} \nabla\left(\frac{f_{i}(u)+u^{T} z_{i}}{g_{i}(u)-u^{T} v_{i}}\right)+\sum_{j=1}^{m} \mu_{j} \nabla\left(h_{j}(u)+u^{T} w_{j}\right) \\
\quad+\sum_{i=1}^{k} \lambda_{i} \nabla_{p} \bar{H}_{i}(u, p)+\sum_{j=1}^{m} \mu_{j} \nabla_{q} K_{j}(u, q)=0,
\end{array} \\
& \sum_{j=1}^{m} \mu_{j}\left[h_{j}(u)+u^{T} w_{j}+K_{j}(u, r)-r^{T} \nabla_{r} K_{j}(u, r)\right] \geq 0, \\
& \sum_{i=1}^{k} \lambda_{i}\left[\bar{H}_{i}(u, s)-s^{T} \nabla_{s} \bar{H}_{i}(u, s)\right] \geq 0, \\
& z_{i} \in C_{i}, \quad v_{i} \in D_{i}, \quad w_{j} \in E_{j}, \quad i=1,2, \ldots, k, j=1,2, \ldots, m, \\
& \lambda_{i}>0, \quad \mu_{j} \geq 0, \quad i=1,2, \ldots, k, j=1,2, \ldots, m .
\end{aligned}
$$

We now discuss the duality results for the primal-dual pair (MFP) and (MFD).

Theorem 4.1 (Weak duality theorem) Let $x \in X^{0}$ and $(u, z, v, \mu, \lambda, w, p, q, r, s)$ be feasible for (MFD). Suppose that:

(i) $\left(f_{i}(\cdot)+(\cdot)^{T} z_{i}\right)$ and $-\left(g_{i}(\cdot)-(\cdot)^{T} v_{i}\right)$ are higher-order $\left(C, \alpha_{i}, \gamma_{i}, \rho_{i}, d_{i}\right)$-convex at $u$, with respect to $H_{i}, p$, and $s, i=1,2, \ldots, k$,

(ii) $\left(h_{j}(\cdot)+(\cdot)^{T} w_{j}\right)$ is higher-order $\left(C, \xi_{j}, \delta_{j}, \eta_{j}, c_{j}\right)$-convex at $u$, with respect to $K_{j}, q$, and $r, j=1,2, \ldots, m$,

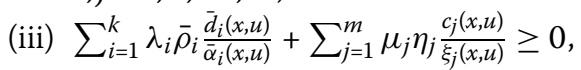

(iv) $\bar{\gamma}_{i}(x, u)=\zeta(x, u)$ and $\xi_{j}(x, u)=\delta_{j}(x, u)=\sigma(x, u), i=1,2, \ldots, k, j=1,2, \ldots, m$, where

$$
\begin{aligned}
& \bar{\alpha}_{i}(x, u)=\left(\frac{g_{i}(x)-x^{T} v_{i}}{g_{i}(u)-u^{T} v_{i}}\right) \alpha_{i}(x, u), \quad \bar{\rho}_{i}=\rho_{i}\left(1+\frac{f_{i}(u)+u^{T} z_{i}}{g_{i}(u)-u^{T} v_{i}}\right), \\
& \bar{d}_{i}(x, u)=\left(\frac{d_{i}(x, u)}{g_{i}(x)-x^{T} v_{i}}\right) .
\end{aligned}
$$

Then the following cannot hold:

$$
\frac{f_{i}(x)+S\left(x \mid C_{i}\right)}{g_{i}(x)-S\left(x \mid D_{i}\right)} \leq \frac{f_{i}(u)+u^{T} z_{i}}{g_{i}(u)-u^{T} v_{i}}, \quad \text { for all } i=1,2, \ldots, k,
$$


and

$$
\frac{f_{r}(x)+S\left(x \mid C_{r}\right)}{g_{r}(x)-S\left(x \mid D_{r}\right)}<\frac{f_{r}(u)+u^{T} z_{r}}{g_{r}(u)-u^{T} v_{r}}, \quad \text { for some } r=1,2, \ldots, k
$$

Proof Suppose that (9) and (10) hold, then using $\frac{\lambda_{i}}{\bar{\alpha}_{i}(x, u)}>0, x^{T} z_{i} \leq S\left(x \mid C_{i}\right), x^{T} v_{i} \leq S\left(x \mid D_{i}\right)$, $i=1,2, \ldots, k$, we have

$$
\sum_{i=1}^{k} \frac{\lambda_{i}}{\bar{\alpha}_{i}(x, u)}\left(\frac{f_{i}(x)+x^{T} z_{i}}{g_{i}(x)-x^{T} v_{i}}-\frac{f_{i}(u)+u^{T} z_{i}}{g_{i}(u)-u^{T} \nu_{i}}\right)<0 .
$$

From hypothesis (i) and Theorem 3.1, $\left(\frac{f_{i}(\cdot)+(\cdot)^{T} z_{i}}{g_{i}(\cdot)-(\cdot)^{T} v_{i}}\right)$ is higher-order $\left(\bar{C}, \bar{\alpha}_{i}, \bar{\gamma}_{i}, \bar{\rho}_{i}, \bar{d}_{i}\right)$-convex at $u$ with respect to $\bar{H}_{i}, p$, and $s, i=1,2, \ldots, k$, therefore

$$
\begin{gathered}
\frac{1}{\bar{\alpha}_{i}(x, u)}\left(\frac{f_{i}(x)+x^{T} z_{i}}{g_{i}(x)-x^{T} v_{i}}-\frac{f_{i}(u)+u^{T} z_{i}}{g_{i}(u)-u^{T} v_{i}}\right) \\
\geq \bar{C}_{x, u}\left(\nabla\left(\frac{f_{i}(u)+u^{T} z_{i}}{g_{i}(u)-u^{T} v_{i}}\right)+\nabla_{p} \bar{H}_{i}(u, p)\right) \\
+\frac{1}{\bar{\gamma}_{i}(x, u)}\left[\bar{H}_{i}(u, s)-s^{T} \nabla_{s} \bar{H}_{i}(u, s)\right]+\frac{\bar{\rho}_{i} \bar{d}_{i}(x, u)}{\bar{\alpha}_{i}(x, u)},
\end{gathered}
$$

and by the higher-order $\left(C, \xi_{j}, \delta_{j}, \eta_{j}, c_{j}\right)$-convex of $\left(h_{j}(\cdot)+(\cdot)^{T} w_{j}\right)$ at $u$ with respect to $K_{j}, q$, and $r, j=1,2, \ldots, m$, we have

$$
\begin{aligned}
& \frac{1}{\xi_{j}(x, u)}\left[h_{j}(x)+x^{T} w_{j}-h_{j}(u)-u^{T} w_{j}\right] \\
& \quad \geq C_{x, u}\left(\nabla\left(h_{j}(u)+u^{T} w_{j}\right)+\nabla_{q} K_{j}(u, q)\right) \\
& \quad+\frac{1}{\delta_{j}(x, u)}\left[K_{j}(u, r)-r^{T} \nabla_{r} K_{j}(u, r)\right]+\frac{\eta_{j} c_{j}(x, u)}{\xi_{j}(x, u)} .
\end{aligned}
$$

Let $\tau=\sum_{i=1}^{k} \lambda_{i} \frac{\left(f_{i}(u)+u^{T} z_{i}+g_{i}(u)-u^{T} v_{i}\right)}{\left(g_{i}(u)-u^{T} v_{i}\right)^{2}}+\sum_{j=1}^{m} \mu_{j}>0$.

Multiply (12) by $\frac{\lambda_{i}}{\tau}$ and (13) by $\frac{\mu_{j}}{\tau}$ and add them, to get

$$
\begin{aligned}
\sum_{i=1}^{k} \frac{\lambda_{i}}{\tau \bar{\alpha}_{i}(x, u)}\left(\frac{f_{i}(x)+x^{T} z_{i}}{g_{i}(x)-x^{T} v_{i}}-\frac{f_{i}(u)+u^{T} z_{i}}{g_{i}(u)-u^{T} v_{i}}\right) \\
\quad+\sum_{j=1}^{m} \frac{\mu_{j}}{\tau \xi_{j}(x, u)}\left[h_{j}(x)+x^{T} w_{j}-h_{j}(u)-u^{T} w_{j}\right] \\
\geq \sum_{i=1}^{k} \frac{\lambda_{i}}{\tau} \frac{\left(f_{i}(u)+u^{T} z_{i}+g_{i}(u)-u^{T} v_{i}\right)}{\left(g_{i}(u)-u^{T} v_{i}\right)^{2}} C_{x, u}\left[\left(\frac{1}{f_{i}(u)+u^{T} z_{i}+g_{i}(u)-u^{T} v_{i}}\right)\right. \\
\quad \times\left(\nabla\left(\frac{f_{i}(u)+u^{T} z_{i}}{g_{i}(u)-u^{T} v_{i}}\right)+\left(\frac{1}{g_{i}(u)-u^{T} v_{i}}+\frac{f_{i}(u)+u^{T} z_{i}}{\left(g_{i}(u)-u^{T} v_{i}\right)^{2}}\right) \nabla_{p} H_{i}(u, p)\right) \\
\left.\quad+\sum_{i=1}^{k} \frac{1}{\tau} \frac{1}{\lambda_{i}} \frac{1}{\bar{\gamma}_{i}(x, u)}\left(\frac{f_{i}(u)+u^{T} z_{i}}{g_{i}(u)-u^{T} v_{i}}\right)\left(H_{i}(u, s)-s^{T} \nabla_{s} H_{i}(u, s)\right)\right]
\end{aligned}
$$




$$
\begin{aligned}
& +\sum_{i=1}^{k} \frac{\lambda_{i}}{\tau} \frac{\rho_{i} d_{i}(x, u)}{\alpha_{i}(x, u)}\left(\frac{1}{g_{i}(u)-u^{T} v_{i}}+\frac{f_{i}(u)+u^{T} z_{i}}{\left(g_{i}(u)-u^{T} v_{i}\right)^{2}}\right) \\
& +\sum_{j=1}^{m} \frac{\mu_{j}}{\tau} C_{x, u}\left(\nabla\left(h_{j}(u)+u^{T} w_{j}\right)+\nabla_{q} K_{j}(u, q)\right) \\
& +\sum_{j=1}^{m} \frac{\mu_{j}}{\tau} \frac{1}{\delta_{j}(x, u)}\left[K_{j}(u, r)-r^{T} \nabla_{r} K_{j}(u, r)\right]+\sum_{j=1}^{m} \frac{\mu_{j}}{\tau} \frac{\eta_{j} c_{j}(x, u)}{\xi_{j}(x, u)} .
\end{aligned}
$$

Further, using convexity on $C$, (6)-(8), and hypotheses (iii)-(iv), we have

$$
\sum_{i=1}^{k} \frac{\lambda_{i}}{\tau \bar{\alpha}_{i}(x, u)}\left(\frac{f_{i}(x)+x^{T} z_{i}}{g_{i}(x)-x^{T} v_{i}}-\frac{f_{i}(u)+u^{T} z_{i}}{g_{i}(u)-u^{T} v_{i}}\right)+\sum_{i=1}^{m} \frac{\mu_{j}}{\tau \sigma(x, u)}\left[h_{j}(x)+x^{T} w_{j}\right] \geq 0 .
$$

Since $x$ is a feasible solution of (MFP), it follows that

$$
\sum_{i=1}^{k} \frac{\lambda_{i}}{\bar{\alpha}_{i}(x, u)}\left(\frac{f_{i}(x)+x^{T} z_{i}}{g_{i}(x)-x^{T} v_{i}}-\frac{f_{i}(u)+u^{T} z_{i}}{g_{i}(u)-u^{T} v_{i}}\right) \geq 0 .
$$

This contradicts (11). Hence we have the result.

Theorem 4.2 (Strong duality theorem) Assume $\bar{u}$ is an efficient solution of (MFP) and let the Slater constraint qualification be satisfied on X. Also, if

$$
\begin{aligned}
& \bar{H}(\bar{u}, 0)=0, \quad K(\bar{u}, 0)=0, \quad \nabla_{p} \bar{H}(\bar{u}, 0)=0, \quad \nabla_{q} K(\bar{u}, 0)=0, \\
& \nabla_{s} \bar{H}(\bar{u}, 0)=0, \quad \nabla_{r} K(\bar{u}, 0)=0,
\end{aligned}
$$

then there exist $0<\bar{\lambda} \in R^{k}, \bar{\mu} \in R^{m}, \bar{z}_{i} \in R^{n}, \bar{v}_{i}$, and $\bar{w}_{j} \in R^{n}, i=1,2, \ldots, k, j=1,2, \ldots, m$, such that $(\bar{u}, \bar{z}, \bar{v}, \bar{\mu}, \bar{\lambda}, \bar{w}, \bar{p}=0, \bar{q}=0, \bar{s}=0, \bar{r}=0)$ is a feasible solution of (MFD) and the objective function values of (MFP) and (MFD) are equal. Furthermore, if the conditions of Theorem 4.1 hold for all feasible solutions of (MFP) and each feasible solution $\left(u^{\prime}, z^{\prime}, v^{\prime}, \mu^{\prime}, \lambda^{\prime}, w^{\prime}, p^{\prime}=0, q^{\prime}=0, r^{\prime}=0, s^{\prime}=0\right)$ of (MFD), then $(\bar{u}, \bar{z}, \bar{v}, \bar{\mu}, \bar{\lambda}, \bar{w}, \bar{p}=0, \bar{q}=0$, $\bar{r}=0, \bar{s}=0)$ is an efficient solution of (MFD).

Proof Assume $\bar{u}$ is an efficient solution of (MFP) and the Slater constraint qualification is satisfied on $X$. Then, from Theorem 3.4, there exist $\bar{\lambda}_{i}>0, \bar{\mu} \in R^{m}, \bar{z}_{i} \in R^{n}, \bar{v}_{i} \in R^{n}$, and $\bar{w}_{j} \in R^{n}, i=1,2, \ldots, k, j=1,2, \ldots, m$, such that

$$
\begin{aligned}
& \sum_{i=1}^{k} \bar{\lambda}_{i} \nabla\left(\frac{f_{i}(\bar{u})+\bar{u}^{T} \bar{z}_{i}}{g_{i}(\bar{u})-\bar{u}^{T} \bar{v}_{i}}\right)+\sum_{j=1}^{m} \bar{\mu}_{j} \nabla\left(h_{j}(\bar{u})+\bar{u}^{T} \bar{w}_{j}\right)=0, \\
& \sum_{j=1}^{m} \bar{\mu}_{j} \nabla\left(h_{j}(\bar{u})+\bar{u}^{T} \bar{w}_{j}\right)=0, \\
& \bar{u}^{T} \bar{z}_{i}=S\left(\bar{u} \mid C_{i}\right), \quad i=1,2, \ldots, k, \\
& \bar{u}^{T} \bar{v}_{i}=S\left(\bar{u} \mid D_{i}\right), \quad i=1,2, \ldots, k, \\
& \bar{u}^{T} \bar{w}_{j}=S\left(\bar{u} \mid E_{j}\right), \quad j=1,2, \ldots, m,
\end{aligned}
$$




$$
\begin{array}{ll}
\bar{z}_{i} \in C_{i}, & \bar{v}_{i} \in D_{i}, \quad \bar{w}_{j} \in E_{j}, \quad i=1,2, \ldots, k, j=1,2, \ldots, m, \\
\bar{\lambda}_{i}>0, & \bar{\mu}_{j} \geq 0, \quad i=1,2, \ldots, k, j=1,2, \ldots, m .
\end{array}
$$

Thus, $(\bar{u}, \bar{z}, \bar{v}, \bar{\mu}, \bar{\lambda}, \bar{w}, \bar{p}=0, \bar{q}=0, \bar{r}=0, \bar{s}=0)$ is feasible for (MFD) and from (16)-(18), the objective function values of (MFP) and (MFD) are equal.

We now show that $(\bar{u}, \bar{z}, \bar{v}, \bar{\mu}, \bar{\lambda}, \bar{w}, \bar{p}=0, \bar{q}=0, \bar{r}=0, \bar{s}=0)$ is an efficient solution of (MFD). If not, then there exists $\left(u^{\prime}, z^{\prime}, v^{\prime}, \mu^{\prime}, \lambda^{\prime}, w^{\prime}, p^{\prime}, q^{\prime}, r^{\prime}, s^{\prime}\right)$ of (MFD) such that

$$
\frac{f_{i}(\bar{u})+\bar{u}^{T} \bar{z}_{i}}{g_{i}(\bar{u})-\bar{u}^{T} \bar{v}_{i}} \leq \frac{f_{i}\left(u^{\prime}\right)+u^{\prime T} z_{i}^{\prime}}{g_{i}\left(u^{\prime}\right)-u^{\prime T} v_{i}^{\prime}}, \quad i=1,2, \ldots, k
$$

and

$$
\frac{f_{r}(\bar{u})+\bar{u}^{T} \bar{z}_{r}}{g_{r}(\bar{u})-\bar{u}^{T} \bar{v}_{r}}<\frac{f_{r}\left(u^{\prime}\right)+u^{\prime T} z_{r}^{\prime}}{g_{r}\left(u^{\prime}\right)-u^{\prime T} v_{r}^{\prime}}, \quad \text { for some } r=1,2, \ldots, k
$$

This contradicts the weak duality theorem. Hence we have the result.

Theorem 4.3 (Strict converse duality theorem) Let $x$ be a feasible solution for (MFP) and $(u, z, v, \mu, \lambda, w, p, q, r, s)$ be feasible for (MFD). Suppose that:

(i) $\left(f_{i}(\cdot)+(\cdot)^{T} z_{i}\right)$ and $-\left(g_{i}(\cdot)-(\cdot)^{T} v_{i}\right)$ are higher-order strictly $\left(C, \alpha_{i}, \gamma_{i}, \rho_{i}, d_{i}\right)$-convex at $u$, with respect to $H_{i}, p$, and $s, i=1,2, \ldots, k$,

(ii) $\left(h_{j}(\cdot)+(\cdot)^{T} w_{j}\right)$ is higher-order $\left(C, \xi_{j}, \delta_{j}, \eta_{j}, c_{j}\right)$-convex at $u$, with respect to $K_{j}, q$, and $r, j=1,2, \ldots, m$,

(iii) $\bar{\gamma}_{i}(x, u)=\zeta(x, u)$ and $\xi_{j}(x, u)=\delta_{j}(x, u)=\sigma(x, u), i=1,2, \ldots, k, j=1,2, \ldots, m$,

(iv) $\sum_{i=1}^{k} \lambda_{i} \bar{\rho}_{i}{\overline{d_{i}(x, u)}}_{\bar{\alpha}_{i}(x, u)}+\sum_{j=1}^{m} \mu_{j} \eta_{j} \frac{c_{j}(x, u)}{\xi_{j}(x, u)} \geq 0$,

(v) $\sum_{i=1}^{k} \frac{\lambda_{i}}{\bar{\alpha}_{i}(x, u)}\left(\frac{f_{i}(x)+x^{T} z_{i}}{g_{i}(x)-x^{T} v_{i}}-\frac{f_{i}(u)+u^{T} z_{i}}{g_{i}(u)-u^{T} v_{i}}\right) \leq 0$, where

$$
\begin{aligned}
& \bar{\alpha}_{i}(x, u)=\left(\frac{g_{i}(x)-x^{T} v_{i}}{g_{i}(u)-u^{T} v_{i}}\right) \alpha_{i}(x, u), \quad \bar{\rho}_{i}=\rho_{i}\left(1+\frac{f_{i}(u)+u^{T} z_{i}}{g_{i}(u)-u^{T} v_{i}}\right), \\
& \bar{d}_{i}(x, u)=\left(\frac{d_{i}(x, u)}{g_{i}(x)-x^{T} v_{i}}\right) .
\end{aligned}
$$

Then $x=u$.

Proof Suppose that $x \neq u$ and exhibit a contradiction. Let $(u, z, v, \mu, \lambda, w, p, q, r, s)$ be feasible for (MFD), then

$$
\begin{aligned}
C_{x, u}(0)= & C_{x, u}\left(\sum_{i=1}^{k} \lambda_{i} \nabla\left(\frac{f_{i}(u)+u^{T} z_{i}}{g_{i}(u)-u^{T} \nu_{i}}\right)+\sum_{j=1}^{m} \mu_{j} \nabla\left(h_{j}(u)+u^{T} w_{j}\right)\right. \\
& \left.+\sum_{i=1}^{k} \lambda_{i} \nabla_{p} \bar{H}_{i}(u, p)+\sum_{j=1}^{m} \mu_{j} \nabla_{q} K_{j}(u, q)\right)=0 .
\end{aligned}
$$


Since $\left(\frac{f_{i}(\cdot)+(\cdot \cdot)^{T} z_{i}}{g_{i}(\cdot)-(\cdot)^{T} v_{i}}\right)$ is higher-order strictly $\left(\bar{C}, \bar{\alpha}_{i}, \bar{\gamma}_{i}, \bar{\rho}_{i}, \bar{d}_{i}\right)$-convex at $u$ with respect to $\bar{H}_{i}, p$, and $s$, we have

$$
\begin{aligned}
& \frac{1}{\bar{\alpha}_{i}(x, u)}\left(\frac{f_{i}(x)+x^{T} z_{i}}{g_{i}(x)-x^{T} v_{i}}-\frac{f_{i}(u)+u^{T} z_{i}}{g_{i}(u)-u^{T} v_{i}}\right) \\
& >\bar{C}_{x, u}\left(\nabla\left(\frac{f_{i}(u)+u^{T} z_{i}}{g_{i}(u)-u^{T} v_{i}}\right)+\nabla_{p} \bar{H}_{i}(u, p)\right) \\
& \quad+\frac{1}{\bar{\gamma}_{i}(x, u)}\left[\bar{H}_{i}(u, s)-s^{T} \nabla_{s} \bar{H}_{i}(u, s)\right]+\frac{\bar{\rho}_{i} \bar{d}_{i}(x, u)}{\bar{\alpha}_{i}(x, u)} .
\end{aligned}
$$

Let $\tau=\sum_{i=1}^{k} \lambda_{i} \frac{\left(f_{i}(u)+u^{T} z_{i}+g_{i}(u)-u^{T} v_{i}\right)}{\left(g_{i}(u)-u^{T} v_{i}\right)^{2}}+\sum_{j=1}^{m} \mu_{j}>0$.

Multiplying by $\frac{\lambda_{i}}{\tau}$ in the above inequality, we get

$$
\begin{aligned}
\frac{\lambda_{i}}{\tau}\left[\frac{1}{\bar{\alpha}_{i}(x, u)}\left(\frac{f_{i}(x)+x^{T} z_{i}}{g_{i}(x)-x^{T} v_{i}}-\frac{f_{i}(u)+u^{T} z_{i}}{g_{i}(u)-u^{T} v_{i}}\right)\right. \\
>\bar{C}_{x, u}\left(\nabla\left(\frac{f_{i}(u)+u^{T} z_{i}}{g_{i}(u)-u^{T} v_{i}}\right)+\nabla_{p} \bar{H}_{i}(u, p)\right) \\
\left.\quad+\frac{1}{\bar{\gamma}_{i}(x, u)}\left[\bar{H}_{i}(u, s)-s^{T} \nabla_{s} \bar{H}_{i}(u, s)\right]+\frac{\bar{\rho}_{i} \bar{d}_{i}(x, u)}{\bar{\alpha}_{i}(x, u)}\right] .
\end{aligned}
$$

By the higher-order $\left(C, \xi_{j}, \delta_{j}, \eta_{j}, c_{j}\right)$-convex of $\left(h_{j}(\cdot)+(\cdot)^{T} w_{j}\right)$ at $u$ with respect to $K_{j}, q$, and $r, j=1,2, \ldots, m$, we obtain

$$
\begin{aligned}
\frac{1}{\xi_{j}(x, u)}\left[h_{j}(x)+x^{T} w_{j}-h_{j}(u)-u^{T} w_{j}\right] \geq & C_{x, u}\left(\nabla\left(h_{j}(u)+u^{T} w_{j}\right)+\nabla_{q} K_{j}(u, q)\right) \\
& +\frac{1}{\delta_{j}(x, u)}\left[K_{j}(u, r)-r^{T} \nabla_{r} K_{j}(u, r)\right]+\frac{\eta_{j} c_{j}(x, u)}{\xi_{j}(x, u)} .
\end{aligned}
$$

It follows that

$$
\begin{aligned}
& \frac{\mu_{j}}{\tau}\left[\frac{1}{\xi_{j}(x, u)}\left[h_{j}(x)+x^{T} w_{j}-h_{j}(u)-u^{T} w_{j}\right]\right. \\
& \geq C_{x, u}\left(\nabla\left(h_{j}(u)+u^{T} w_{j}\right)+\nabla_{q} K_{j}(u, q)\right) \\
& \left.\quad+\frac{1}{\delta_{j}(x, u)}\left[K_{j}(u, r)-r^{T} \nabla_{r} K_{j}(u, r)\right]+\frac{\eta_{j} c_{j}(x, u)}{\xi_{j}(x, u)}\right] .
\end{aligned}
$$

Taking summation over $i$ in (22) and over $j$ in (23), we get

$$
\begin{aligned}
& \sum_{i=1}^{k} \frac{\lambda_{i}}{\tau \bar{\alpha}_{i}(x, u)}\left(\frac{f_{i}(x)+x^{T} z_{i}}{g_{i}(x)-x^{T} v_{i}}-\frac{f_{i}(u)+u^{T} z_{i}}{g_{i}(u)-u^{T} v_{i}}\right) \\
& \quad+\sum_{j=1}^{m} \frac{\mu_{j}}{\tau \xi_{j}(x, u)}\left[h_{j}(x)+x^{T} w_{j}-h_{j}(u)-u^{T} w_{j}\right] \\
& >\sum_{i=1}^{k} \frac{\lambda_{i}}{\tau} \bar{C}_{x, u}\left(\nabla\left(\frac{f_{i}(u)+u^{T} z_{i}}{g_{i}(u)-u^{T} v_{i}}\right)+\nabla_{p} \bar{H}_{i}(u, p)\right) \\
& \quad+\sum_{i=1}^{k} \frac{\lambda_{i}}{\tau} \frac{1}{\bar{\gamma}_{i}(x, u)}\left[\bar{H}_{i}(u, s)-s^{T} \nabla_{s} \bar{H}_{i}(u, s)\right]
\end{aligned}
$$




$$
\begin{aligned}
& +\sum_{i=1}^{k} \frac{\lambda_{i}}{\tau} \frac{\bar{\rho}_{i} \bar{d}_{i}(x, u)}{\bar{\alpha}_{i}(x, u)}+\sum_{j=1}^{m} \frac{\mu_{j}}{\tau} C_{x, u}\left(\nabla\left(h_{j}(u)+u^{T} w_{j}\right)+\nabla_{q} K_{j}(u, q)\right) \\
& +\sum_{j=1}^{m} \frac{\mu_{j}}{\tau} \frac{1}{\delta_{j}(x, u)}\left[K_{j}(u, r)-r^{T} \nabla_{r} K_{j}(u, r)\right]+\sum_{j=1}^{m} \frac{\mu_{j}}{\tau} \frac{\eta_{j} c_{j}(x, u)}{\xi_{j}(x, u)} .
\end{aligned}
$$

Further, using convexity on $C$ and (21), we obtain

$$
\begin{aligned}
\sum_{i=1}^{k} & \frac{\lambda_{i}}{\tau \bar{\alpha}_{i}(x, u)}\left(\frac{f_{i}(x)+x^{T} z_{i}}{g_{i}(x)-x^{T} v_{i}}-\frac{f_{i}(u)+u^{T} z_{i}}{g_{i}(u)-u^{T} v_{i}}\right) \\
& +\sum_{j=1}^{m} \frac{\mu_{j}}{\tau \xi_{j}(x, u)}\left[h_{j}(x)+x^{T} w_{j}-h_{j}(u)-u^{T} w_{j}\right] \\
> & \sum_{i=1}^{k} \frac{\lambda_{i}}{\tau} \frac{1}{\bar{\gamma}_{i}(x, u)}\left[\bar{H}_{i}(u, s)-s^{T} \nabla_{s} \bar{H}_{i}(u, s)\right]+\sum_{j=1}^{m} \frac{\mu_{j}}{\tau} \frac{1}{\delta_{j}(x, u)}\left[K_{j}(u, r)-r^{T} \nabla_{r} K_{j}(u, r)\right] \\
& +\sum_{i=1}^{k} \frac{\lambda_{i}}{\tau} \frac{\bar{\rho}_{i} \bar{d}_{i}(x, u)}{\bar{\alpha}_{i}(x, u)}+\sum_{j=1}^{m} \frac{\mu_{j}}{\tau} \frac{\eta_{j} c_{j}(x, u)}{\xi_{j}(x, u)} .
\end{aligned}
$$

This, together with (7)-(8) and hypotheses (iii)-(iv), shows

$$
\sum_{i=1}^{k} \frac{\lambda_{i}}{\tau \bar{\alpha}_{i}(x, u)}\left(\frac{f_{i}(x)+x^{T} z_{i}}{g_{i}(x)-x^{T} v_{i}}-\frac{f_{i}(u)+u^{T} z_{i}}{g_{i}(u)-u^{T} v_{i}}\right)+\sum_{i=1}^{m} \frac{\mu_{j}}{\tau \sigma(x, u)}\left[h_{j}(x)+x^{T} w_{j}\right]>0 .
$$

Since $x$ is a feasible solution of (MFP), it follows that

$$
\sum_{i=1}^{k} \frac{\lambda_{i}}{\bar{\alpha}_{i}(x, u)}\left(\frac{f_{i}(x)+x^{T} z_{i}}{g_{i}(x)-x^{T} v_{i}}-\frac{f_{i}(u)+u^{T} z_{i}}{g_{i}(u)-u^{T} v_{i}}\right)>0
$$

This contradicts the hypothesis (v). Hence the proof is completed.

\section{Conclusions}

In this article, we consider a class of fractional programming problem having $k$-objectives in which each numerator and denominator of the objective function is nondifferentiable in terms of the support function of a compact convex set. The important property that the ratio of higher-order $(C, \alpha, \gamma, \rho, d)$-convex function is also a higher-order $(\bar{C}, \bar{\alpha}, \bar{\gamma}, \bar{\rho}, \bar{d})$ convex function is obtained. We also derive sufficient optimality conditions for an efficient solution for this problem. Furthermore, a higher-order Mond-Weir type dual is formulated and appropriate duality relations are obtained under higher-order $(C, \alpha, \gamma, \rho, d)$-convexity assumptions.

\section{Competing interests}

The authors declare that they have no competing interests.

Authors' contributions

All authors contributed equally to the writing of this paper. All authors read and approved the final manuscript.

\section{Author details}

'Department of Mathematics, Indian Institute of Technology, Roorkee, 247 667, India. ${ }^{2}$ Department of Mathematics, University of Tabuk, Tabuk, Saudi Arabia. 


\section{Acknowledgements}

The authors are thankful to the reviewers for their valuable and constructive suggestions. The authors are also grateful to Deanship of Scientific Research (DSR), University of Tabuk, Saudi Arabia under Grant No. S-0131-1436, for their support to carry out this work.

Received: 9 June 2015 Accepted: 29 October 2015 Published online: 09 November 2015

\section{References}

1. Mangasarian, OL: Second and higher-order duality in nonlinear programming. J. Math. Anal. Appl. 51, 607-620 (1975)

2. Chen, X: Higher-order symmetric duality in nondifferentiable multiobjective programming problems. J. Math. Anal. Appl. 290, 423-435 (2004)

3. Kassem, MA: Higher-order symmetric duality in vector optimization problem involving generalized cone-invex functions. Appl. Math. Comput. 209, 405-409 (2009)

4. Suneja, SK, Srivastava, MK, Bhatia, M: Higher order duality in multiobjective fractional programming with support functions. J. Math. Anal. Appl. 347, 8-17 (2008)

5. Ying, G: Higher-order symmetric duality for a class of multiobjective fractional programming problems. J. Inequal. Appl. 2012, 142 (2012)

6. Yuan, $\mathrm{DH}$, Chinchuluun, A, Pardalos, PM: Nondifferentiable minimax fractional programming problems with $(C, \alpha, \rho, d)$-convexity. J. Optim. Theory Appl. 185, 185-199 (2006)

7. Chinchuluun, A, Yuan, D, Pardalos, PM: Optimality conditions and duality for nondifferentiable multiobjective fractional programming with generalized convexity. Ann. Oper. Res. 154, 133-147 (2007)

8. Long, XJ: Optimality conditions and duality for nondifferentiable multiobjective programming problems with $(C, \alpha, \rho, d)$-convexity. J. Optim. Theory Appl. 148, 197-208 (2011)

9. Hanson, MA: On sufficiency of the Kuhn-Tucker conditions. J. Math. Anal. Appl. 80, 545-550 (1981)

10. Gupta, SK, Danger, D, Ahmad, I: On second order duality for nondifferentiable minimax fractional programming problems involving type-I functions. ANZIAM J. 55, 479-494 (2014)

11. Pandey, S: Duality for multiobjective fractional programming involving generalized $\eta$-convex functions. Opsearch 28 , 31-43 (1991)

12. Gulati, TR, Saini, H: Higher-order $(F, \alpha, \beta, \rho, d)$-convexity and its application in fractional programming. Eur. J. Pure Appl. Math. 4, 266-275 (2011)

13. Liang, ZA, Huang, HX, Pardalos, PM: Optimality conditions and duality for a class of nonlinear fractional programming problem. J. Optim. Theory Appl. 110, 611-619 (2001)

14. Gupta, SK, Kailey, N, Sharma, MK: Multiobjective second-order nondifferentiable symmetric duality involving $(F, \alpha, \rho, d)$-convex function. J. Appl. Math. Inform. 28, 1395-1408 (2010)

15. Chen, $X$ : Sufficiency conditions and duality for a class of multiobjective fractional programming problems with higher-order $(F, \alpha, \rho, d)$-convexity. J. Appl. Math. Comput. 28, 107-121 (2008)

16. Egudo, RR: Multiobjective fractional duality. Bull. Aust. Math. Soc. 37, 367-378 (1988)

17. Geoffrion, AM: Proper efficiency and the theory of vector maximization. J. Math. Anal. Appl. 22, 618-630 (1968)

18. Gulati, TR, Geeta: Duality in nondifferentiable multiobjective fractional programming problem with generalized invexity. J. Appl. Math. Comput. 35, 103-118 (2011)

\section{Submit your manuscript to a SpringerOpen ${ }^{\circ}$ journal and benefit from:}

- Convenient online submission

Rigorous peer review

- Immediate publication on acceptance

- Open access: articles freely available online

- High visibility within the field

- Retaining the copyright to your article 